\title{
HIV and Aging: Double Stigma
}

\author{
Monique J. Brown ${ }^{1,2,3,4}$ (D) Oluwafemi Adeagbo ${ }^{2,5,6}$ \\ Accepted: 23 February 2021 / Published online: 12 March 2021 \\ (C) The Author(s), under exclusive licence to Springer Nature Switzerland AG 2021
}

\begin{abstract}
Purpose of Review HIV and ageism continue to be key public health challenges in the USA and globally. Older people living with HIV may experience intersectional stigma resulting from HIV and ageism. The current review summarizes the scientific literature and focuses on social isolation and lack of social support as key factors in experiencing HIV-related and aging-related stigma.

Recent Findings Social isolation and social support are key social determinants of health, which may have a bidirectional relationship with HIV-related stigma and ageism. Stigmatization may also result in health care providers not paying enough attention to the mental health and sexual health needs of older adults.

Summary Current research suggests that the intersection of HIV-related stigma and ageism is a complex issue. Future research should focus on the design and feasibility of implementing stigma reduction interventions addressing HIV-related stigma and ageism.
\end{abstract}

Keywords HIV and aging $\cdot$ Older adults $\cdot$ Stigma $\cdot$ Ageism $\cdot$ HIV stigma $\cdot$ HIV-related stigma

\section{Introduction}

The definition of stigma has evolved with time. Stigma was defined as "not quite human" or "tainted" by Goffman in 1963 where being different may evoke hatred or animosity from

This article is part of the Topical Collection on Epidemiology of Aging

Monique J. Brown

brownm68@mailbox.sc.edu

1 Department of Epidemiology and Biostatistics, Arnold School of Public Health, University of South Carolina, Columbia, SC 29208, USA

2 South Carolina SmartState Center for Healthcare Quality, Arnold School of Public Health, University of South Carolina, Columbia, SC, USA

3 Rural and Minority Health Research Center, Arnold School of Public Health, University of South Carolina, Columbia, SC, USA

4 Office for the Study on Aging, Arnold School of Public Health, University of South Carolina, Columbia, SC, USA

5 Department of Health Promotion, Education, and Behavior, Arnold School of Public Health, University of South Carolina, Columbia, SC, USA

6 Department of Sociology, Faculty of Humanities, University of Johannesburg, Johannesburg, South Africa others [1]. In 1989, Campbell defined stigmatization as labeling or branding, or developing signs that "appear to justify being labeled or branded" [2]. In other words, a person is devalued due to being different [3]. Dudley defined stigma as "stereotypes or negative views attributed to a person or groups of people when their characteristics or behaviors are viewed as different from or inferior to societal norms" $[4,5]$.

There are consequences of stigma and these include "stereotyping, dehumanization, and dependence" [3]. Stereotyping allows for generalizations to be treated as truths [6], which can result in devaluation and dehumanization [3] of the individual or group. Dehumanization may create dependence in individuals who perceive an inability to surpass the labels [3] that are linked to their stigmatized identity. Dependence may occur as "learned helplessness" when individuals feel ashamed, expect failure, or have feelings of powerlessness [7].

One illness that continues to be stigmatized is HIV/AIDS, which continues to be a key public health challenge among older adults. Even though incidence rates have declined among older adults, approximately one in six new HIV diagnoses were among adults aged 50 and older in the USA in 2018 [8]. Older adults who are living with HIV may undergo HIV-related and age-related stigma [9]. Approximately seven in ten older adults living with HIV experience HIV-related and age-related stigma [9]. Currently, there is paucity of data 
on older adults living with HIV, experience of stigma, social support, and their sexual health needs across the globe. Following the calls for attention on older PLWH in public health $[10-13,14 \bullet \cdot]$, we examine two salient aspects of the three-fold stigma [15], ageism, and HIV-related stigma to tease out important areas for future research and investments for older adults living with HIV.

\section{Age-Related Stigma/Ageism}

Ageism is defined as the "negative attitudes, stereotypes, and behaviors that are directed towards older adults based solely on their perceived age" $[16,17]$, and has been found in a variety of places including at work, or in health care settings [17]. It has also been defined as the stereotyping of and discriminating against people due to their older age [18], in addition to avoidance of dealing with their challenges [2]. Ageism consists of three facets: cognitive, affective, and behavioral [19]. It is important to note that implicit attitudes and stereotypes do not only apply to race but are also applicable to aging [19] in a variety of areas such as housing, work, healthcare, and among family and friends [17]. Implicit ageism is said to be more prevalent than $[20,21]$ but has similar effects to explicit ageism [22].

Ageism can be based on the perceiver's perspective as well as the perspective of older adults [17] and can be expressed at the micro-, macro-, and meso-levels [19]. Indeed, internalized ageism was a major theme emerging from interviews of adults aged 50 and older living with HIV [9]. The perceptions of older adults are complex and may include negative as well as positive perspectives [17]. Positive stereotypes tend to be associated with the "young-old" (aged 55-64) while negative stereotypes tend to be linked with the "old-old" (aged 75 and older) [17]. The main determinant of internalized ageism was found to be health status while determinants of ageism directed towards others was quality of contact with older adults, and the presentation of older adults to others, whether positive or negative [19].

\section{Ageism and Adverse Outcomes}

Ageism has been shown to be associated with a variety of outcomes such as mortality and physical and/or functional health including frailty [23], subjective accelerated aging (the rate at which someone feels he/she/they are aging) [24], mental health, cognition, overall quality of life, and health behaviors [25••]. The operationalization of ageism also plays a significant role in these associations: age stereotype [25••], selfperceptions of aging [25, 26], and age discrimination [25, 26]. Significant moderators in the association between ageism and health included self-relevance, and demographic characteristics such as age, race/ethnicity, gender, and perceived social status [25••]. In addition, the link between ageism and subjective accelerated aging is moderated by self-age awareness and other- age awareness [24]. Ageism has been found to be negatively associated with measures of well-being with the religiosity level of the country serving as a moderator in this relationship [27]. The higher the religiosity level of the country is, the less evident is the relationship between ageism and well-being. Ageism was also associated with dementia-related anxiety or the fear of developing Alzheimer's disease and related dementias [28]. Nevertheless, at the structural level, ageism has been shown to be linked to cultural aspects such as greater levels of masculinity and long-term orientation ("the extent to which a culture programs its members to accept delayed gratification of their material, social, and emotional needs") [29]. Ageism has shown to be significantly reduced when the percentage of older adults is greater and with greater increases in life expectancy [30].

Stereotypes such as forgetfulness and mental incompetence are also salient, which can result in negative behaviors towards older adults [17]. These negative behaviors can also impact healthcare for older adults and specifically prevent access to mental health care [31]. Aging sexual stigma may result in a lack of sexual health care for older adults [32]. Nevertheless, cognitive behavioral therapy has been shown to reduce ageism among health care providers [33].

\section{Ageism, Social Isolation, and Social Support}

Many older adults experience social isolation [34], which is a major public health challenge [35]. Older adults are more likely to experience social isolation due to role transitions, changes in physical and mental health, and views of society [35]. Social isolation may signify a lack of social support, and has been linked to negative impacts on income, ability to deal with elder abuse, and successful aging [34]. Indeed, social isolation and social support have emerged as key social determinants of health [35]. Lack of social support was found to be associated with greater anxiety towards aging [36]. Loneliness, which may result from social isolation may impact physical and mental health outcomes such as depression, cognition, and also increases the risk of mortality [37••]. Ezra and colleagues proposed three mechanisms via which ageism is a contributing factor to loneliness and social isolation: (1) social rejection, which may result in avoidance and withdrawal from social participation; (2) internalization of stereotypes which suggest that being older signifies inevitable loneliness; and (3) discrimination that accentuates social exclusion of older adults [37••]. Age-related stigma or ageism can exacerbate social isolation and decrease social support for older adults.

\section{HIV-Related Stigma}

People living with HIV (PLWH) now live longer because of recent advances in antiretroviral therapy (ART). Although ART access was initially restricted to high-income countries, large global investments into HIV programs has made access 
to ART possible for PLWH, especially those in sub-Saharan Africa (SSA) - a region with the highest number of PLWH $[10,38]$. These investments had significant impact on the drastic reduction in AIDS-related mortality as well as the improved life expectancy of PLWH [10, 14••, 39, 40]. PLWH are more likely to have a normal life expectancy or 20 to 50 years following HIV infection [41, 42]. Like the United States and other high-income countries, half of PLWH in SSA are 50 years and older [13, 14, 41, 43, 44•]. In fact, an estimated 10 million PLWH in SSA will be above age 50 years by 2040 [45]. However, little is known about aging with HIV and associated stigma. Older adults aging with HIV face various challenges such as accelerated aging, stigma, frailty, and other comorbidities $[10,43]$. To date, the global response to HIV predominantly focuses on mothers, children, adolescents' girls, and young women while ignoring the needs of older adults living with HIV [13, 14••].

Stigma has been established in several studies as the main driver of the HIV epidemic as well as a major barrier to the HIV care cascade [42, 46-48]. This is further complicated by "ageism" especially among older adults living with HIV [49]. Stigma reduction or eradication is key to achieving the ambitious zero new HIV infections by 2030 by UNAIDS [42, 50]. Despite the calls to eradicate stigma, it remains a major barrier in HIV care with very few interventions aimed at reducing stigma among PLWH [51-53]. Systematic reviews of HIV-related stigma found that despite the improvement in stigma research, there is a lack of data on stigma interventions across the world [53-, 54•]. There is still a need for further research on HIV-related stigma and interventions to achieve the ambitious UNAIDS 9595-95 strategy of ending the AIDS epidemic.

\section{Isolation of Older Adults in Global Response to HIV: a "Brewing Epidemic"}

The United Nations high-level meeting on AIDS and noncommunicable diseases held in June and September 2011, respectively, highlighted the importance of combining HIV services with maternal and child health services but ignored the growing evidence on aging with HIV $[13,14 \bullet \cdot]$. A decade later, little attention has been paid to this issue that requires urgent attention. Older adults are often not seen as an HIV risk group due to the misconceptions that they are not sexually active, thereby ignoring their sexual health needs in the global response to HIV. HIV prevention programs hardly focus on older adults [10, 13, 55, 56]. Misconceptions, lack of tailored HIV programs, and tailored health resources make it difficult for older adults to seek medical help given the assumption that they are sexually inactive. In fact, healthcare providers are less likely to discuss HIV testing or other related issues with older adults $[14 \bullet \bullet, 56]$. Mutevedzi \& Newell concluded that despite the high number of HIV infections among older adults in SSA, they are largely excluded in the HIV response [55]. Similarly, in the United States, it was reported since the early 90s that healthcare providers were not paying attention to HIV infection in older adults because they assumed that older adults were not sexually active, and, therefore, not at risk of contracting HIV [13, 57-59].

Studies have shown that providers were less likely to associate HIV-related symptoms to HIV acquisition in older adults because of the perceived assumptions that older people are not at risk of contracting HIV [55, 60, 61]. In contrast, data from South Africa, United States, and United Kingdom found that a substantial number of older adults aged 50 years and above tested positive for HIV [55, 62-64]. Contrary to the misconceptions that older adults are not sexually active, literature has consistently shown that older adults aged 50 years and above are sexually active [55, 65]. Data from Asia [66], Africa [55], Europe [67], and the USA [68] show that older adults, particularly men, engage in sexual activity. Studies from highincome countries showed limited HIV knowledge among older adults when compared to younger people [13, 69]. There is consistency across different settings that older adults are less likely to use a condom, thereby increasing their risk of contracting or transmitting HIV especially in SSA where agedisparate sexual relationships are common $[13,55,70,71]$. A recent review of global HIV and aging revealed that little is known about HIV knowledge and sexual practices of older adults across the world [56]. This evidence reveals the need to prioritize the sexual reproductive health needs of older adults if we are to achieve the ambitious UNAIDS 95-95-95 strategy. This is because they are more likely to engage in risky sexual behaviors, acquire, and transmit HIV without adequate HIV knowledge. Addressing the needs of older PLWH will require a rethinking and some investments such as human resources (e.g., geriatric clinicians), strengthening the health systems, and clinical infrastructure $[10,14 \bullet \bullet, 43]$.

\section{Informal or Formal Social Support? Exploring HIV-related Stigma Among Older Adults Living With HIV}

HIV-related stigma affects older PLWH as well as their relationships with family and friends [46, 72, 73]. Literature has consistently shown that older PLWH are more likely to experience higher levels of stress due to social isolation [44••, 46, 72, 74]. Similarly, fear of stigma and non-disclosure of HIV status could affect older PLWH willingness to access social support [46, 72]. Studies have shown that supportive environment and social relationships can build self-esteem and self-efficacy of older adults living with HIV [46, 47]. Also, research from SSA has shown that access to HIV services and social support impacted the overall wellbeing of PLWH [10, 47]. Similarly, research from the United States showed the importance of support networks, 
self-reliance, coping strategies, and mental health on healthy aging among PLWH $[42,72,75]$. However, the description of healthy aging gives credence to individual roles and responsibilities within family and social networks in SSA, thereby putting pressure on older PLWH [76, 77].

Most studies on social support of chronic disease selfmanagement focused on informal sources of social support such as care provided by family or friends [44••, 78, 79]. However, little is known about formal social support such as help from healthcare providers. Evidence has shown that some older adults may rely on formal sources to provide both instrumental and emotional support. A study concluded that many older adults living with HIV may be isolated from support from family members or friends and must depend on formal support (e.g., healthcare providers) for care [80]. In contrast, social support needs of older PLWH are often ignored by formal sources of support as described above given that both "older age" and "HIV" are highly stigmatized. Thus, both formal (e.g., healthcare providers) and informal (e.g., family) social support have significant roles to play in the psychological, physiological, and health outcomes of older adults living with HIV.

\section{Conclusion: Way Forward}

This review highlights the need for a global response to address the intersection of HIV and age-related stigma. Indeed, one of the perceived barriers to receiving social support from family friends among older adults living with HIV was ageism [81]. Decades of HIV research across the world demonstrated priority interventions and several gains (e.g., the introduction of ART and preexposure prophylaxis (PrEP) in reducing AIDS-related mortality and by keeping the epidemic under control). Despite these gains, older adults living with HIV are constantly excluded from HIV programs. Of importance will be formative research on the experiences (including their experience of stigma and coping strategies) of older people living with HIV as well as their sexual health needs. It is often assumed that older people (irrespective of their HIV status) are not sexually active; therefore, most current health interventions or programs exclude older adults. Preliminary data would subsequently help in developing culturally acceptable tailored interventions for their needs in different settings. To sustain the gains of global investment in the HIV care cascade, there is a need to prioritize stigma reduction interventions and sexual health needs of older adults living with HIV.

\section{Compliance with Ethical Standards}

Conflict of Interest Monique J. Brown and Oluwafemi Adeagbo declare that they have no conflict of interest.
Human and Animal Rights and Informed Consent This article does not contain any studies with human or animal subjects performed by any of the authors.

\section{References}

Papers of particular interest, published recently, have been highlighted as:

- Of importance

•- Of major importance

1. Goffman E. Stigma; Notes on the management of spoiled identity. New York: Jason Aronson; 1963.

2. Campbell RJ. Psychiatric Dictionary. New York: Oxford University Press; 1989.

3. Herrick CA, Pearcey LG, Ross C. Stigma and ageism: compounding influences in making an accurate mental health assessment. Nurs Forum. 1997;32(3):21-6. https://doi.org/10.1111/j. 1744-6198.1997.tb00206.x.

4. Dudley JR. Confronting stigma within the services system. Soc Work. 2000;45(5):449-55. https://doi.org/10.1093/sw/45.5.449.

5. Ahmedani BK. Mental health stigma: society, individuals, and the profession. J Soc Work Values Ethics. 2011;8(2):41-416.

6. Ainlay SC, Becker G, Coleman L. M. The dilemma of difference: a multidisciplinary view of stigma. New York: Plenum Press; 1986.

7. Ebersole P, Hess P. Development of gerontology. In: Ebersole P, Hess P, editors. Toward healthy aging. 4th ed. St. Louis: MosbyYearbook; 1997. p. 521-62.

8. HIV and older Americans [https://www.cdc.gov/hiv/group/age/ olderamericans/index.html]

9. Emlet CA. "You're awfully old to have this disease": experiences of stigma and ageism in adults 50 years and older living with HIV/ AIDS. Gerontologist. 2006;46(6):781-90. https://doi.org/10.1093/ geront/46.6.781.

10. Siedner MJ. Aging, health, and quality of life for older people living with HIV in sub-Saharan Africa: a review and proposed conceptual framework. J Aging Health. 2019;31(1):109-38. https://doi.org/10. $1177 / 0898264317724549$.

11. Green A. NIH project focuses on integration of HIV and NCD care. Lancet. 2016;388(10054):1869. https://doi.org/10.1016/S01406736(16)31846-3.

12. High KP, Brennan-Ing M, Clifford DB, Cohen MH, Currier J, Deeks SG, et al. HIV and aging: state of knowledge and areas of critical need for research. A report to the NIH Office of AIDS Research by the HIV and Aging Working Group. J Acquir Immune Defic Syndr. 2012;60:S1-S18. https://doi.org/10.1097/ QAI.0b013e31825a3668.

13. Negin J, Mills EJ, Bärnighausen T. Aging with HIV in Africa: the challenges of living longer. AIDS 2012;26(0 1):S1. 10.1097/ QAD.0b013e3283560f54

14.• Till Bärnighausen M, MIA JN. HIV and aging - preparing for the challenges ahead. N Engl J Med. 2012;366(14):1270. https://doi. org/10.1056/NEJMp1113643 This is one of the few manuscripts that highlight the challenges of HIV and aging in both low and middle income countries and high income countries. It sets the tone for some of the issues discussed in this paper.

15. Brown MJ, Serovich JM, Kimberly JA, Umasabor-Bubu O. Disclosure and self-efficacy among HIV-positive men who have sex with men: a comparison between older and younger adults. AIDS Patient Care STDs. 2015;29(11):625-33. https://doi.org/10. 1089/apc.2015.0133. 
16. Butler RN. Age-ism: another form of bigotry. Gerontologist. 1969;9(4):243-6. https://doi.org/10.1093/geront/9.4_part_1.243.

17. Richeson JA, Sheltin JN. A social psychological perspective on the stigmatization of older adults. In: Carstensen LLH, C.R, editors. When I'm 64 committee on aging frontiers in social psychology, personality, and adult developmental psychology. Washington, D. C.: The National Academies Press; 2006.

18. Bahr SRT. An overview of gerontological nursing. In: Hogstel MO, editor. Nursing care of the older adult. Albany, NY: Delmar; 1994. p. 2-25.

19. Marques S, Mariano J, Mendonça J, De Tavernier W, Hess M, Naegele L, et al. Determinants of ageism against older adults: a systematic review. Int J Environ Res Public Health. 2020;17(7): 2560. https://doi.org/10.3390/ijerph17072560.

20. Hummert ML. Age stereotypes and aging. In: Schaie KW, editor. Handbook of the psychology of aging. Willis, SL: Elsevier; 2011. p. 249-62.

21. Levy B, Banaji MR. Implicit ageism. In: Nelson TD, editor. Ageism: Stereotyping and prejudice against older persons. Cambridge, MA: The MIT Press; 2002. p. 49-75.

22. Horton SBJ, Pearce GW, Deakin JM. On the malleability of performance: implications for seniors. J Appl Gerontol. 2008;27:446-65.

23. Ye B, Gao J, Fu H, Chen H, Dong W, Gu M. How does ageism influence frailty? A preliminary study using a structural equation model. BMC Geriatr. 2020;20(1):422. https://doi.org/10.1186/ s12877-020-01749-8.

24. Bergman YS, Palgi Y. Ageism, personal and others' perceptions of age awareness, and their interactive effect on subjective accelerated aging. J Appl Gerontol. 2020 [Epub ahead of print]: 073346482096720. https://doi.org/10.1177/0733464820967209.

25.• Hu RX, Luo M, Zhang A, Li LW. Associations of ageism and health: a systematic review of quantitative observational studies. Res Aging 2020 [Epub ahead of print]. https://doi.org/10.1177/ 0164027520980130 . This systematic review examined the relationship between ageism and health outcomes which were categorized as "disease, mortality, physical/functional health, mental health, cognitive function, quality of life and health behavior", and made a significant contribution to this paper.

26. Choi EY, Kim Y, Chipalo E, Lee HY. Does perceived ageism widen the digital divide? And does it vary by gender? Gerontologist. 2020;60(7):1213-23. https://doi.org/10.1093/ geront/gnaa066.

27. Kim HH, Jung JH. Ageism, religiosity, and wellbeing among older adults: evidence from the European Social Survey (ESS4). Res Aging. 2020 [Epub ahead of print]:016402752095363. https://doi. org/10.1177/0164027520953632.

28. Yun SW, Maxfield M. Correlates of dementia-related anxiety: selfperceived dementia risk and ageism. Educ Gerontol. 2020;46(9): 563-74. https://doi.org/10.1080/03601277.2020.1790103.

29. Ng R, Lim WJ. Ageism linked to culture, not demographics: evidence from an 8-billion-word corpus across 20 countries. J Gerntol B Psychol Sci Soc Sci. 2020 [Epub ahead of print]. https://doi.org/ 10.1093/geronb/gbaa181.

30. Officer A, Thiyagarajan JA, Schneiders ML, Nash P, de la FuenteNúñez V. Ageism, healthy life expectancy and population ageing: how are they related? Int J Environ Res Public Health. 2020;17(9): 3159. https://doi.org/10.3390/ijerph17093159.

31. Carter R. Mental illnesses and the elderly: Double stigma, double jeopardy. In: The Fifth Annual Rosalynn Carter Symposium on Mental Health Policy. Atlanta: Emory University School of Medicine, Department of Psychiatry; 1989.

32. Syme ML, Cohn TJ. Examining aging sexual stigma attitudes among adults by gender, age, and generational status. Aging
Ment Health. 2016;20(1):36-45. https://doi.org/10.1080/ 13607863.2015 .1012044$.

33. Rababa M, Alhawatmeh H, Al Ali N, Kassab M. Testing the effectiveness of cognitive behavioral therapy in relieving nurses' ageism toward older adults: a randomized controlled trial. Cognit Ther Res. 2020 [Epub ahead of print]. https://doi.org/10.1007/s10608-02010167-4.

34. Guruge S, Sidani S, Wang L, Sethi B, Spitzer D, Walton-Roberts $\mathrm{M}$, et al. Understanding social network and support for older immigrants in Ontario, Canada: protocol for a mixed-methods study. JMIR Aging. 2019;2(1):e12616. https://doi.org/10.2196/12616.

35. Smith ML, Steinman LE, Casey EA. Combatting social isolation among older adults in a time of physical distancing: the COVID-19 social connectivity paradox. Front Public Health. 2020;8:403. https://doi.org/10.3389/fpubh.2020.00403.

36. Ramirez LP, Palacios-Espinosa X. Stereotypes about old age, social support, aging anxiety and evaluations of one's own health. Journal of Social Issues. 2016;72(1):47-68. https://doi.org/10.1111/josi.12155.

37.• Shiovitz-Ezra S, Shemesh J, McDonnell/Naughton M. Pathways from ageism to loneliness. In: Ayalon L, Tesch-Römer C, editors. Contemporary Perspectives on Ageism. Cham: Springer International Publishing; 2018. p. 131-47. This chapter highlights three potential pathways from ageism to loneliness which included social rejection, internalization of stereotypes and discrimination.

38. Bendavid E, Ford N, Mills EJ. HIV and Africa's elderly: the problems and possibilities. AIDS. 2012;26:S85-91. https://doi.org/10. 1097/QAD.0b013e3283558513.

39. Bor J, Herbst AJ, Newell M-L, Bärnighausen T. Increases in adult life expectancy in rural South Africa: valuing the scale-up of HIV treatment. Science. 2013;339(6122):961-5. https://doi.org/10. 1126/science.1230413.

40. Reniers G, Blom S, Calvert C, Martin-Onraet A, Herbst AJ, Eaton JW, et al. Trends in the burden of HIV mortality after roll-out of antiretroviral therapy in KwaZulu-Natal, South Africa: an observational community cohort study. Lancet HIV. 2017;4(3):e113-21. https://doi.org/10.1016/S2352-3018(16)30225-9.

41. Durand M, Chartrand-Lefebvre C, Baril J-G, Trottier S, Trottier B, Harris M, et al. The Canadian HIV and aging cohort studydeterminants of increased risk of cardio-vascular diseases in HIVinfected individuals: rationale and study protocol. BMC Infect Dis. 2017;17(1):1-10. https://doi.org/10.1186/s12879-017-2692-2.

42. Nyblade L, Mbuya-Brown RJ, Ezekiel MJ, Addo NA, Sabasaba AN, Atuahene K, et al. A total facility approach to reducing HIV stigma in health facilities: implementation process and lessons learned. AIDS. 2020;34(Suppl1):S93-S102. https://doi.org/10. 1097/qad.0000000000002585.

43. Wing EJ. HIV and aging. Int J Infect Dis. 2016;53:61-8. https://doi. org/10.1016/j.ijid.2016.10.004.

44. Warren-Jeanpiere L, Dillaway H, Hamilton P, Young M, Goparaju L. Life begins at 60: identifying the social support needs of African American women aging with HIV. J Health Care Poor Underserved. 2017;28(1):389-405. https://doi.org/10.1353/hpu. 2017.0030 This paper highlighted that older African American women needed support from healthcare providers, family members and other people living with HIV.

45. Hontelez JA, de Vlas SJ, Baltussen R, Newell M-L, Bakker R, Tanser $\mathrm{F}$, et al. The impact of antiretroviral treatment on the age composition of the HIV epidemic in sub-Saharan Africa. AIDS. 2012;26(Suppl 1(0 1)):S19-30. https://doi.org/10.1097/QAD. 0b013e3283558526.

46. Qiao S, Ingram L, Deal ML, Li X, Weissman SB. Resilience resources among African American women living with HIV in 
Southern United States. AIDS. 2019;33(Supp11):S35-44. https:// doi.org/10.1097/QAD.0000000000002179.

47. Adamu A, Mchunu G, Naidoo JR. Stress and resilience among women living with HIV in Nigeria. Afr J Prim Health Care Fam Med. 2019;11(1):e1-6. https://doi.org/10.4102/phcfm.v11i1.2046.

48. Stahlman S, Grosso A, Ketende S, Sweitzer S, Mothopeng T, Taruberekera N, et al. Depression and social stigma among MSM in Lesotho: implications for HIV and sexually transmitted infection prevention. AIDS Behav. 2015;19(8):1460-9. https://doi.org/10. 1007/s10461-015-1094-y.

49. Ageism and older adults with HIV: a source of health disparities [https://www.apa.org/news/press/releases/2017/08/older-adultshiv.pdf]

50. Derose KP, Griffin BA, Kanouse DE, Bogart LM, Williams MV, Haas AC, et al. Effects of a pilot church-based intervention to reduce HIV stigma and promote HIV testing among African Americans and Latinos. AIDS Behav. 2016;20(8):1692-705. https://doi.org/10.1007/s10461-015-1280-y.

51. Pulerwitz J, Michaelis A, Weiss E, Brown L, Mahendra V. Reducing HIV-related stigma: lessons learned from Horizons research and programs. Public Health Rep. 2010;125(2):272-81. https://doi.org/10.1177/003335491012500218.

52. Li L, Wu Z, Liang L-J, Lin C, Guan J, Jia M, et al. Reducing HIVrelated stigma in health care settings: a randomized controlled trial in China. Am J Public Health. 2013;103(2):286-92. https://doi.org/ 10.2105/AJPH.2012.300854

53. Ikeda DJ, Nyblade L, Srithanaviboonchai K, Agins BD. A quality improvement approach to the reduction of HIV-related stigma and discrimination in healthcare settings. BMJ Global Health. 2019;4(3):e001587. https://doi.org/10.1136/bmjgh-2019-001587 This paper highlights a quality improvement approach to reducing HIV-related stigma in healthcare settings which continues to be a challenge at present.

54. Rao D, Elshafei A, Nguyen M, Hatzenbuehler ML, Frey S, Go VF. A systematic review of multi-level stigma interventions: state of the science and future directions. BMC Med. 2019;17(1):1-11. https:// doi.org/10.1186/s12916-018-1244-y This systematic review examined the importance of stigma interventions, which will be crucial to addressing the issues outlined in this review.

55. Mutevedzi PC, Newell M-L. A missing piece in the puzzle: HIV in mature adults in sub-Saharan Africa. Future Virol. 2011;6(6):75567.

56. Sprague C, Brown SM. Local and global HIV aging demographics and research. In: Brennan-Ing M, DeMarco DF, editors. HIV and Aging, vol. 42: Karger Publishers; 2017. p. 1-10. https://doi.org/10. $1159 / 000448532$.

57. Gropper EI. Can your nursing home resident possibly have AIDS? Geriatr Nurs. 1990;11(5):240-2. https://doi.org/10.1016/s01974572(05)80465-3

58. Scura KW, Whipple B. Older adults as an HIV-positive risk group. J Gerontol Nurs. 1990;16(2):6-9. https://doi.org/10.3928/00989134-19900201-04.

59. Gaeta TJ, LaPolla C, Melendez E. AIDS in the elderly: New York City vital statistics. The J Emerg Med. 1996;14(1):19-23. https:// doi.org/10.1016/0736-4679(95)02038-1.

60. El-Sadr W, Gettler J. Unrecognized human immunodeficiency virus infection in the elderly. Arch Intern Med. 1995;155(2):184-6.

61. Whipple B, Scura KW. The overlooked epidemic: HIV in older adults. Am J Nurs. 1996;96(2):23-9.

62. Wallrauch C, Bärnighausen T, Newell M-L. HIV prevalence and incidence in people 50 years and older in rural South Africa. S Afr Med J. 2010;100(12):812-3. https://doi.org/10.7196/samj.4181.
63. Simone MJ, Appelbaum J. HIV in older adults. Geriatrics. 2008;63(12):6-12.

64. Smith RD, Delpech VC, Brown AE, Rice BD. HIV transmission and high rates of late diagnoses among adults aged 50 years and over. AIDS. 2010;24(13):2109-15. https://doi.org/10.1097/QAD. $0 \mathrm{~b} 013 \mathrm{e} 32833 \mathrm{c} 7 \mathrm{~b} 9 \mathrm{c}$.

65. Nicolosi A, Laumann EO, Glasser DB, Moreira ED Jr, Paik A, Gingell C. Sexual behavior and sexual dysfunctions after age 40: the global study of sexual attitudes and behaviors. Urology. 2004;64(5):991-7. https://doi.org/10.1016/j.urology.2004.06.055.

66. Wong SYS, Leung JCS, Woo J. Sexual activity, erectile dysfunction and their correlates among 1,566 older Chinese men in Southern China. J Sex Med. 2009;6(1):74-80. https://doi.org/10. 1111/j.1743-6109.2008.01034.x.

67. Moreira E, Hartmann U, Glasser D, Gingell C. A population survey of sexual activity, sexual dysfunction and associated helpseeking behavior in middle-aged and older adults in Germany. Eur J Med Res. 2005;10(10):434-43.

68. Lindau ST, Schumm LP, Laumann EO, Levinson W, O'Muircheartaigh CA, Waite LJ. A study of sexuality and health among older adults in the United States. N Engl J Med. 2007;357(8):762-74.

69. Abel T, Werner M. HIV risk behaviour of older persons. The European Journal of Public Health. 2003;13(4):350-2. https://doi. org/10.1056/NEJMoa067423.

70. Schick V, Herbenick D, Reece M, Sanders SA, Dodge B, Middlestadt SE, et al. Sexual behaviors, condom use, and sexual health of Americans over 50: implications for sexual health promotion for older adults. J Sex Med. 2010;7:315-29. https://doi.org/10. 1111/j.1743-6109.2010.02013.x.

71. Negin J, Cumming RG. HIV infection in older adults in subSaharan Africa: extrapolating prevalence from existing data. Bull World Health Org. 2010;88:847-53. https://doi.org/10.2471/BLT. 10.076349 .

72. Subramaniam S, Camacho LM, Carolan MT, López-Zerón G. Resilience in low-income African American women living and aging with HIV. J Women Aging. 2017;29(6):543-50. https://doi.org/ 10.1080/08952841.2016.1256735.

73. Dale SK, Cohen MH, Kelso GA, Cruise RC, Weber KM, Watson $\mathrm{C}$, et al. Resilience among women with HIV: impact of silencing the self and socioeconomic factors. Sex Roles. 2014;70(5-6):22131. https://doi.org/10.1007/s11199-014-0348-x.

74. Heckman TG, Heckman B, Kochman A, Sikkema KJ, Suhr J, Goodkin K. Psychological symptoms among persons 50 years of age and older living with HIV disease. Aging Ment Health. 2002;6(2):121-8. https://doi.org/10.1080/13607860220126709a.

75. DeGrezia MG, Scrandis D. Successful coping in urban, community-dwelling older adults with HIV. J Assoc Nurses AIDS Care. 2015;26(2):151-63. https://doi.org/10.1016/j.jana. 2014.11.008

76. Ogunmefun C, Gilbert L, Schatz E. Older female caregivers and HIV/AIDS-related secondary stigma in rural South Africa. J Cross Cult Gerontol. 2011;26(1):85-102. https://doi.org/10.1007/s10823010-9129-3.

77. Schatz E, Gilbert L. "My legs affect me a lot. ... I can no longer walk to the forest to fetch firewood": challenges related to health and the performance of daily tasks for older women in a high HIV context. Health Care Women Int. 2014;35(7-9):771-88. https://doi. org/10.1080/07399332.2014.900064.

78. Williams SW, Dilworth-Anderson P. Systems of social support in families who care for dependent African American elders. Gerontologist. 2002;42(2):224-36. https://doi.org/10.1093/geront/ 42.2.224. 
79. Becker G, Newsom E. Resilience in the face of serious illness among chronically ill African Americans in later life. J Gerontol B Psychol Sci Soc Sci. 2005;60(4):S214-23. https://doi.org/10. 1093/geronb/60.4.s214.

80. Shippy RA, Karpiak SE. Perceptions of support among older adults with HIV. Res Aging. 2005;27(3):290-306. https://doi.org/10. $1177 / 0164027504273780$.
81. Schrimshaw EW, Siegel K. Perceived barriers to social support from family and friends among older adults with HIV/AIDS. J Health Psychol. 2003;8(6):738-52. https://doi.org/10.1177/ 13591053030086007.

Publisher's Note Springer Nature remains neutral with regard to jurisdictional claims in published maps and institutional affiliations. 